\title{
Globe
}

Revue internationale d'études québécoises

\section{Repenser le Québec dans un Canada multinational. Pour un modèle fonctionnel de la citoyenneté}

\section{François Rocher}

Volume 1, numéro 1, 1998

URI : https://id.erudit.org/iderudit/1000102ar

DOI : https://doi.org/10.7202/1000102ar

Aller au sommaire du numéro

Éditeur(s)

Globe, Revue internationale d'études québécoises

ISSN

1481-5869 (imprimé)

1923-8231 (numérique)

Découvrir la revue

Citer cet article

Rocher, F. (1998). Repenser le Québec dans un Canada multinational. Pour un modèle fonctionnel de la citoyenneté. Globe, 1(1), 77-113.

https://doi.org/10.7202/1000102ar
Résumé de l'article

Pour plusieurs, la fragmentation des identités et des appartenances se présente comme une menace à l'établissement d'une citoyenneté commune et partagée et à la protection équivalente des droits individuels sur un territoire donné. Cet article soutient, en se référant particulièrement au cas canadien, que cette crainte est alimentée par une vision jacobine de l'État qui s'avère problématique dans les États multinationaux, pouvant même conduire à son éclatement. Deux éléments retiennent l'attention. D’abord, la présence de référents identitaires particuliers n'est pas nécessairement contradictoire avec l'établissement d'une citoyenneté qui subsume les différences, à la condition que celle-ci accepte et reconnaisse qu'il existe plusieurs niveaux

d'appartenance. C'est pourquoi dans le cas d'États plurinationaux, et plus particulièrement dans ceux qui s'inspirent des principes du fédéralisme, la citoyenneté peut être fonctionnelle plutôt que fondée sur une dynamique d'homogénéisation des différences. Ensuite, cette citoyenneté fonctionnelle, dans un espace politique qui se veut démocratique et inclusif, doit se fonder sur un ensemble de droits fondamentaux devant être considérés à la lumière de la préservation de la communauté première d'appartenance.
Tous droits réservés @ Globe, Revue internationale d’études québécoises, 1998

Tous droits réserves $\mathbb{0}$ Globe, Revue internationale d'études québecoises, 1998

Ce document est protégé par la loi sur le droit d'auteur. L’utilisation des services d'Érudit (y compris la reproduction) est assujettie à sa politique d'utilisation que vous pouvez consulter en ligne.

https://apropos.erudit.org/fr/usagers/politique-dutilisation/ 


\title{
Repenser le Québec dans un Canada multinational. Pour un modèle fonctionnel de la citoyenneté ${ }^{1}$
}

\author{
François Rocher \\ Carleton University
}

Pour certains, la fragmentation des identités et des appartenances se présente comme une menace à l'établissement d'une citoyenneté commune et partagée et à la protection équivalente des droits individuels sur un territoire donné. Le projet politique canadien a cherché à redéfinir les paramètres de l'appartenance au Canada sur des bases essentiellement individuelles. La Charte canadienne des droits et libertés et la reconfiguration symbolique de la citoyenneté qui l'accompagne reposent sur la prémisse de la primauté de l'individu sur la collectivité. Ce faisant, l'existence du Québec comme communauté politique singulière, tout comme c'est le cas des communautés autochtones au Canada, ne peut que se fondre (sinon être incorporée) dans cette nouvelle représentation du Canada. Celle-ci ne laisse aucune place à des représentations de la citoyenneté qui ouvriraient la porte à des aménagements

1 Cette réflexion s'inscrit dans le cadre des travaux menés par le Groupe de recherche sur les sociétés plurinationales (GRSP), financé par le Fonds pour les chercheurs et l'aide à la recherche (FCAR). Ce texte est une version remaniée d'une communication présentée dans le cadre d'un atelier portant sur les droits fondamentaux et la citoyenneté, organisé conjointement par l'Institut international de sociologie juridique d'Onati et la Commission des droits de la personne du Québec, et qui s'est tenu à Onati, Espagne, en mai 1998.

François Rocher, «Repenser le Québec dans un Canada multinational. Pour un modèle fonctionnel de la citoyennetém, Globe. Revue intermationale d'itudes québécoises, vol. 1, no 1, 1998. 
respectueux de la diversité qui caractérise pourtant la réalité politique et sociale canadienne. Il existerait une tension entre les principes universels devant être le fondement des démocraties constitutionnelles et les demandes de nature particulariste émanant des communautés nationales, celles-ci cherchant à préserver leur intégrité et leur identité propres (Habermas, 1995, 256).

Cette façon de poser le problème ne dissocie pas deux éléments qui ne sont pourtant pas de même nature : à prime abord, la citoyenneté renvoie au membership politique, alors que la question de l'identité se situe à un autre niveau, celui de l'appartenance à un groupe particulier, de la définition de l'individu en fonction de sa communauté d'appartenance, d'un sentiment plus ou moins ancré, plus ou moins amovible. Les frontières de la citoyenneté ne recoupent pas nécessairement celles de l'identité. Cette distinction nous apparaît être fondamentale à la fois pour mieux appréhender les conditions permettant l'établissement d'une citoyenneté partagée, et pour analyser comment des aménagements particuliers doivent être mis en place afin d'assurer la pérennité des communautés nationales.

Nous soutiendrons, en nous référant particulièrement à la dynamique Québec-Canada, que la crainte de la fragmentation des appartenances est alimentée par une vision jacobine de l'État, qui s'avère problématique dans les États multinationaux (que l'on doit distinguer des États polyethniques), pouvant même conduire à leur éclatement. Deux éléments retiendront notre attention. D'abord, la présence de référents identitaires particuliers n'est pas nécessairement contradictoire avec l'établissement d'une citoyenneté qui subsume les différences, à la condition que celle-ci accepte et reconnaisse qu'il existe plusieurs niveaux d'appartenance. C'est pourquoi nous croyons que, dans le cas d'Etats multinationaux comme le Canada, et plus particulièrement dans ceux qui s'inspirent des principes du 
fédéralisme, la citoyenneté puisse être fonctionnelle plutôt que fondée sur une dynamique d'homogénéisation des différences. Ensuite, nous soutiendrons que cette citoyenneté fonctionnelle, dans un espace politique qui se veut démocratique et inclusif, doit se fonder sur un ensemble de droits fondamentaux devant être considérés à la lumière de la préservation de la communauté première d'appartenance. C'est dans cet esprit que doit être appréhendée la citoyenneté québécoise. Cela ne signifie pas que les libertés fondamentales individuelles soient mises de côté, mais tout simplement qu'elles doivent être mises en juxtaposition avec la nécessité de préserver la communauté au sein de laquelle elles peuvent s'exprimer.

\section{Identité, nationalité et citoyenneté Une clarification conceptuelle}

Identité nationale et citoyenneté sont souvent des termes interchangeables. Ils renvoient à la question fondamentale de savoir ce qui unit les habitants partageant un même espace, qu'il soit politique, culturel, social, ou tout cela à la fois. Partager une identité, c'est déterminer de manière plus ou moins lâche, par le droit d'une part ou le sentiment d'appartenance d'autre part, les conditions d'appartenance au gxoupe. Posée de manière différente, la question est de savoir comment les conditions d'appartenance sont définies. Processus à la fois inclusif et exchusif, l'identification des conditions d'appartenance est le produit d'un rapport social et/ou politique. L'identité d'un citoyen au sein d'un État repose aussi bien sur l'appartenance à un groupe particulier (que ce soit par sa culture, sa langue, sa religion, ses traditions) que sur le fait qu'il participe à une même communauté politique. Pour Gamberale (1997, 37), l'identité nationale est de "nature transcendantale» dans la mesure où ses éléments constitutifs ne sont pas définis politiquement par les citoyens, mais précèdent l'appartenance politique. L'ethnicité, la 
culture, la langue, la religion et les traditions déterminent les frontières de la communauté avant et au-delà de la volonté politique des citoyens. Il s'agit essentiellement d'un phénomène de conscience. C'est dire que la citoyenneté et l'identité politique doivent être comprises comme le résultat du discours politique et de la volonté exprimée par les individus de participer à une association de nature politique. En somme, la citoyenneté renvoie à lidée d'association, alors que la nationalité est basée sur l'idée du sentiment d'appartenance.

Cette distinction entre nation et citoyenneté peut paraître artificielle. La citoyenneté repose sur le modèle de l'association politique. L'espace est ainsi bien délimité. La relation est d'abord et avant tout de nature contractuelle et se trouve balisée par le droit. Les institutions politiques, quant à elles, définissent les mécanismes permettant la participation (ou la nonparticipation) des individus vivant à l'intérieur des frontières politiques. Elles permettent de délimiter l'espace de la délibération publique ainsi que les conditions et les mécanismes à travers lesquels cette délibération est rendue possible. Pour sa part, lidentité nationale est présentée comme un phénomène antérieur à l'appartenance politique. Elle transcende cette dernière. La nation définit un regroupement particulier qui se définit en premier lieu en dehors du politique. L'identité nationale est celle qui se construit historiquement sur la base des caractéristiques que les membres de la nation trouvent pertinentes dans le temps et dans l'espace. Si l'identité nationale est transcendantale, elle se transporte aussi dans l'arène politique. La délibération politique n'est pas sans être teintée par la façon dont les individus se définissent et se reconnaissent. L'espace politique n'existe pas dans un vacuum. La nation interpelle le politique sans s'y réduire.

Se pose donc le problème de la cœxistence entre la nation et la citoyenneté. $\mathrm{Ce}$ problème soulève deux questions importantes : comment est-il possible de concilier les identités 
nationales dans un espace politique qui peut compter plus d'une nation? ; au sein d'une communauté politique (définie comme lieu de délibération publique) multinationale, quelle place est réservée aux membres des groupes ethniques minoritaires qui ne sont pas considérés comme des nations? Le problème est celui de la tension qui existe entre les identités nationale et politique. Une identité politique commune, celle qui est associée à la citoyenneté, ne devrait pas, du moins en théorie, être incompatible avec les autres formes d'identités et d'allégeances (nationales, culturelles, religieuses). De plus, alors que l'identité nationale est le fruit du processus de définition des balises d'appartenance au groupe (en identifiant un nous par rapport à un autre), l'identité politique et la citoyenneté s'inscrivent dans une démarche d'inclusion, parce qu'elles reposent entièrement sur les éléments politiques de l'association (Gamberale, 1997, 38).

\section{De la nation et de l'ethnie}

Dans le vocabulaire des sciences sociales, le terme de "nation» est utilisé de manière indifférenciée pour décrire l'ensemble des institutions politiques (l'État-nation ou la «nation politique» canadienne, américaine, française, espagnole), une communauté «ethnique» particulière (la nation canadienne française, la nation écossaise ou les nations autochtones) ou les procédés politiques conduisant à la création et à la consolidation de la nation. Toutefois, toutes les communautés ne sont pas des nations.

Kymlicka (1995a, 1995b) soutient que la diversité culturelle peut prendre plusieurs formes. La première consiste en la cœxistence de plusieurs nations dans un État particulier, la nation étant définie comme une communauté historique territorialement circonscrite, possédant un ensemble d'institutions assez complet, partageant une langue et une 
histoire propres. Pour Kymlicka, un pays qui abrite plus d'une culture (comprise ici comme nation) n'est pas un État-nation, mais plutôt un État multinational, "où les cultures plus petites forment des "minorités nationales" ou "cultures minoritaires") (1995b, 94). La seconde source de pluralité culturelle provient des mouvements migratoires. Ces divers groupes ethniques constituent des sous-cultures plus ou moins bien intégrées, mais ne peuvent prétendre constituer des cultures complètes comme le sont les cultures dites nationales. Dans cette perspective, un pays peut être à la fois multinational et polyethnique.

Encore ici, il faut distinguer ethnie, définie comme une communauté de langue et de culture, et l'ethnicité, qui renvoie plutôt à la conscience d'appartenir et de partager les caractéristiques propres à une communauté dite ethnique, qui est son expression. Or l'ethnicité n'est pas une construction figée, intangible, contrairement au terme auquel elle est trop souvent associée : celui de race. Il s'agit d'un phénomène social en évolution faisant appel à un processus de construction/déconstruction. Elle ne trouve son expression que dans des phénomènes subjectifs qui sont culturels et peuvent être politiques. C'est pourquoi le sens d'appartenance est essentiel à la constitution d'une communauté ethnique et, plus fondamentalement, à une communauté nationale. Pour Cahen, «il importe de ne pas tout confondre : communauté n'est pas nécessairement ethnicité, le degré de cristallisation, l'étendue du champ sur lequel porte le sentiment identitaire et le facteur-temps étant des critères fondamentauxos (1994, 20). Ainsi, il $\mathrm{y}$ a des phénomènes identitaires qui peuvent être transitoires, notamment dans le cas de minorités ethniques issues de vagues d'immigration récentes, sans qu'on assiste à la consolidation de minorités nationales durables. Celles-ci doivent s'inscrire dans une dynamique de consolidation, de durée historique et de cristallisation d'un sentiment d'appartenance ou, en d'autres termes, d'une identité. Cette 
quête identitaire, lorsqu'elle prend des dimensions politiques, lorsqu'elle s'inscrit dans des rapports de forces et met de l'avant des revendications visant à assurer les bases permettant une durée dans le temps et une reconnaissance implicite ou explicite de la part des autres composantes de l'ensemble politique, assure le passage de l'ethnie à la nation.

L'ethnie n'est pas nécessairement la nation, mais la nation, telle qu'on la définit ici, renvoie nécessairement à l'ethnie et surtout à la dynamique identitaire ancrée dans l'histoire, à l'autoreconnaissance subjective de ce qui lui est propre. Ainsi, il existe une gradation dans la durée et dans la cristallisation du sentiment ethnique (Cahen, 1994, 83). C'est pourquoi, contrairement à une définition immuable de l'ethnie (appréhendée dans le sens de «tace»), cette approche implique qu'un individu puisse changer d'ethnicité au cours de sa vie et qu'une communauté peut revoir les paramètres de son identité (comme en fait foi le passage identitaire des francophones québécois de "Canadiens français» avant les années 1960 à "Québécois»). Finalement, la nation peut être ouverte, dans la mesure où cette communauté de langue et de culture peut chercher à inclure de nouvelles composantes et s'en trouver ainsi transformée, ou fermée, et définir de manière figée les caractéristiques de la nation. Cette distinction, banale en apparence, est pourtant lourde de conséquences politiques, particulièrement dans les États multinationaux et polyethniques.

Bon nombre d'États, que l'on pense au Canada, à l'Espagne, à la Suisse, à la Belgique, sont dans une très large mesure composés de plusieurs communautés nationales. La question qui se pose est celle de la reconnaissance des identités intermédiaires entre le citoyen atomisé et l'État. Il existe une tension réelle entre les identités intermédiaires (quelles soient nationales, culturelles, religieuses, reliées à l'appartenance sexuelle ou à un mode de vie particulier), luniversalisme et le caractère 
apparemment neutre de l'État, appréhendé comme la somme des citoyens qui le composent. A un autre niveau, on peut se demander, au-delà des particularismes, ce qui lie les citoyens au sein d'une communauté politique.

\section{De la citoyenneté}

La citoyenneté peut être comprise comme un sentiment de type communautaire lié à l'habitude de vivre dans le même ensemble institutionnel et à la capacité d'influencer son évolution. Ainsi, la citoyenneté est intimement liée d'une part aux droits individuels, et d'autre part, à l'attachement à une communauté politique particulière. La citoyenneté véhicule un sens d'appartenance.

Pour Kymlicka et Norman (1995, 284), les théories de la citoyenneté renvoient à deux conceptualisations différentes : la citoyenneté comme statut légal permettant une participation pleine et entière dans une communauté politique particulière, et la citoyenneté comme activité souhaitable, où la qualité de la citoyenneté est fonction du degré de participation dans la communauté politique. Ces deux approches ne sont pas contradictoires, mais reposent sur des questionnements de nature différente. Pour sa part, Beiner tient compte de ces deux dimensions. La citoyenneté est présentée comme une forme d'attachement à la communauté politique elle-même. Mais cet attachement implique aussi des pratiques sociales, culturelles, économiques et politiques qui rendent tangible cet attachement et premettent de lui donner corps. Selon lui, "[i]n the ideal case, citizenship is active membership in a political community where the very fact of such membership empowers those included in it to contribute to the shaping of a shared collective destiny) (1992, 105). Dans les États polyethniques et multinationaux, ce sentiment de partager une destinée collective doit transcender les particularismes. On peut 
se demander quelles sont les conditions permettant à cet universalisme de subsumer les différences tout en ne les niant pas, tout en ne mettant pas de l'avant un ensemble de principes qui, bien que s'inscrivant dans les valeurs universelles, accepte les manifestations différenciées à l'endroit de cet attachement. En d'autres termes, l'égalité présumée des citoyens au sein d'une même communauté politique ne doit pas masquer le fait que ceux-ci peuvent avoir un attachement tout aussi profond, sinon plus, envers leur communauté première d'appartenance (qu'elle soit culturelle ou nationale).

Pour Beiner (1995, 12), le problème tient au fait que nous sommes assignés à choisir entre deux approches mutuellement exclusives. D'une part, on retrouve les différentes sortes d'universalisme qui exaltent l'inviolable valeur morale des individus, appréhendés comme des êtres humains atomisés, qui se situent au-dessus et au-delà de toute forme d'identité collective ou civique qui devrait particulariser les êtres humains. Cette vision universaliste est suspicieuse à l'endroit de toute forme de citoyenneté différenciée dans la mesure où cette demière implique une identité primordiale, à la fois exclusive et particulariste. D'autre part, on retrouve les forces du particularisme qui célèbrent et affirment ces formes d'identités collectives, condition permettant de distinguer les ensembles d'individus les uns des autres. Cette citoyenneté qui accepte les particularismes peut alimenter des explosions nationalistes et ethniques qui contribuent en retour à rendre inopérante la citoyenneté, ou le sentiment d'appartenir à une même communauté politique.

Cette tension entre universalisme et particularisme peut-elle être atténuée? N'est-elle pas commune à toutes les sociétés dans la mesure où aucune ne peut faire abstraction du fait que les identités individuelles sont le produit d'une histoire, d'un processus de socialisation, d'une insertion dans une communauté particulière? L'approche libérale met l'accent sur 
l'individu, sur sa capacité de transcender son groupe ou son identité collective, de briser les chaînes d'une identité prédéterminée, de définir ses propres buts. Dans cette perspective, la communauté politique n'est qu'instrumentale, puisqu'elle n'existe que pour permettre aux individus de donner un sens à leur existence. L'approche "communautarienne» met l'accent sur le groupe culturel, ethnique, national, sur la solidarité qui existe entre ceux qui partagent une même histoire et une même tradition, sur la capacité du groupe à conférer une identité aux individus autrement atomisés par les tendances que l'on retrouve au sein des sociétés libérales à déraciner les individus. Cette perspective est tout autant instrumentale dans la mesure où la communauté politique n'existe que pour alimenter une identité collective qui trouve son fondement premier en dehors du politique. Finalement, l'approche républicaine met l'accent sur ce qui lie les citoyens. Ceux-ci ne sont pas seulement atomisés et n'existent pas qu'à travers leur appartenance à un groupe. La communauté politique est un «bien» en elle-même. Les traditions politiques constituent des totalités vivantes qui ne peuvent être réduites aux fins individuelles ou aux objectifs des communautés primordiales. Ainsi, la communauté politique est vue comme l'expression d'une identité civique (Beiner, 1995, 13-15). Pour cet auteur, la demière approche apparait comme supérieure aux deux autres puisqu'elle insiste sur la dynamique politique propre à chaque État. Toutefois, son appréhension de l'approche qu'il qualifie de "communautarienne» est réductrice dans la mesure où elle semble s'opposer aux principes du libéralisme.

Or, le libéralisme n'est pas incompatible avec la reconnaissance du fait qu'il existe une communauté nationale au sein de laquelle doivent s'exercer les droits des citoyens. En d'autres termes, la nation n'absorbe pas les individus. Le nationalisme libéral peut se conjuguer aux valeurs humaines d'égalité, de solidarité et de liberté. Pour Tamir, l'autonomie personnelle et l'appartenance à une communauté sont des alliées 
naturelles. De tels "concepts sont ici perçus cormme des idées complémentaires plutôt qu'opposées, ce qui impliquerait qu'un individu ne peut exister libre de tout contexte, mais que tous peuvent être libres à l'intérieur d'un même contexte.» $(1993,14)$

La citoyenneté se différencie donc du sentiment national tel que nous l'avons préalablement défini - la nation «ethnique» ou "culturelle». Il ne faut donc pas confondre citoyenneté et nationalité. Dans la perspective libérale classique, l'État appartient à tous les citoyens sans distinction religieuse, ethnique, nationale, etc. L'État de droit ne peut être le fondement de l'identité nationale parce que la nation ne peut être "fondée», elle ne peut être le fruit de politiques gouvernementales puisqu'elle est le résultat d'un long processus historique d'auto-reconnaissance qui dépasse les frontières du politique. En ce sens, la nation précède et déborde l'État. On ne peut donc pas définir la nation sur la base du seul critère de la citoyenneté. Opter pour une telle approche représente une négation de l'existence de niveaux d'identification intermédiaires entre le citoyen et l'État. Qui plus est, un tel choix nie l'existence de communautés nationales (cristallisées ou en voie de l'être) au sein d'un même système politique. Favoriser une définition uniquement citoyenne de la nation dans une perspective libérale individualiste, c'est mettre de l'avant un effort d'homogénéisation ethnique en faveur de la forme d'ethnicité dominante, c'est-à-dire celle de la communauté ethnique / nationale dominante. La qualité de la citoyenneté dépend au contraire de la capacité d'exprimer les particularités culturelles auxquelles les citoyens sont attachés et auxquelles ils s'identifient. De plus, l'attachement des citoyens à la unation élective» sera fonction des garanties de liberté et de développement accordées aux «identités nationales» qui la composent, dans la mesure où il s'agit de la meilleure solution dans une conjoncture particulière. 
Dans les États polyethniques et / ou multinationaux, la tentation peut être grande de vouloir créer une «nation» ou un sentiment d'identité nationale. Dans un tel processus, deux approches peuvent être adoptées. La première peut être la négation pure et simple des différences. Au nom d'un ensemble de valeurs, symboles, normes et règles dites universelles qui devraient unir l'ensemble des citoyens, on privilégie le renforcement de la communauté nationale dominante (non-avouée). Les communautés ethniques et / ou nationales minoritaires doivent être intégrées dans le tout. Cette homogénéisation, s'inspirant de la nation politique néojacobine, est recherchée au nom de l'harmonie sociale, de la stabilité politique et de la nécessaire intégration (lire assimilation) des éléments minoritaires au sein d'un tout faussement indifférencié. L'uniformité linguistique et culturelle est essentielle au bon fonctionnement de l'Etat modeme (Gellner, 1981, 140-141). La seconde approche est plus interventionniste. Identifiant la diversité comme un "problème», l'État cherche à créer une «nation» nouvelle qui, tout en reconnaissant les différences, les subsume. Ancrées dans leurs particularismes et aveuglées par eux, les communautés nationales sont incapables de concourir au bien de l'ensemble. Seul l'État «neutre» est en mesure de parler au nom du tout. Cette deuxième approche, bien que reconnaissant les particularismes, ne leur accorde aucune légitimité politique. Les «nations» peuvent être reconnues sociologiquement, mais aucune reconnaissance politique n'en découle au nom de la préservation d'une uniformité des droits devant lier les citoyens. Dans un tel contexte, l'État cherche à créer des mythes fondateurs et des symboles unificateurs, ce qui est particulièrement difficile à imposer dans des États à tradition démocratique et / ou dans les États où les clivages sont nombreux et territorialement bien circonscrits. Dans un tel cas, la tentation peut être forte de combiner les deux approches, laissant aux communautés nationales territorialement constituées le soin d'opérer leur propre processus d'homogénéisation nationale, ouvrant 
potentiellement la porte à des excès de purification ethnique dans des États à faible tradition démocratique.

Ainsi, la question qui se pose dans les États polyethniques / multinationaux est celle de la prise en compte de la diversité, de sa reconnaissance et de sa gestion. C'est ainsi que les problématiques du multiculturalisme et de la diversité s'avèrent particulièrement pertinentes dans la mesure où l'État canadien s'est inspiré de la première problématique, alors que l'État québécois a plutôt privilégié la seconde.

Les notions de multiculturalisme et de pluralité recouvrent elles aussi des significations multiples. Ainsi, le multiculturalisme et la pluralité peuvent être appréhendés comme des faits, des idéologies, des politiques ou des processus. La société canadienne comprend indubitablement plusieurs groupes ethniques et nationaux. En termes abstraits, tous ces groupes méritent d'être considérés sur le même pied. Toutefois, force est d'admettre que toute société a besoin, pour fonctionner, d'un minimum de dénominateurs communs ou de ce que Julien Harvey et Gary Caldwell appelaient une culture publique commune. À l'échelle du Canada, c'est la culture anglosaxonne qui s'est imposée comme culture dominante à travers notamment la langue anglaise, portée originellement par les membres du groupe ethnique d'origine britannique. Au Québec, c'est la culture de tradition française, à travers notamment la langue portée originellement par les membres du groupe ethnique d'origine française, qui constitue celle par laquelle l'intégration est appelée à se réaliser.

En partie pour déclasser le modèle biculturel mis de l'avant par la Commission Laurendeau-Dunton qui devait inspirer un réaménagement du fédéralisme canadien, le gouvernement fédéral a mis de l'avant au début des années 1970 une politique visant à tenir compte du caractère polyethnique de la société canadienne. $\mathrm{La}$ notion de multiculturalisme, néologisme 
typiquement canadien, bien que difficile à définir, renvoie d'abord à un projet politique, à une orientation publique visant à favoriser l'intégration, mais aussi la préservation et le respect des composantes minoritaires au sein de l'ensemble canadien. En d'autres termes, il s'agit d'une politique publique favorisant l'ajustement de la société à la réalité de la diversité ethnique. Au lieu de l'ignorer ou de la répudier, l'État a choisi d'y faire face.

Encore faut-il souligner que le terme multiculturalisme est porteur de significations multiples. Par-dessus tout, il est piégé parce qu'il s'inscrit dans le langage politique et juridique de l'État canadien. Dans ce contexte, il devient difficile de faire la part des choses entre son contenu idéologique et politique (le discours de l'État et les politiques publiques portant sur cette dimension de la réalité), l'utilisation qui en est faite par les porte-parole des groupes ethniques et / ou culturels dans leur rapports avec l'État (le clientélisme ou le corporatisme ethnique), la réalité sociale dont il tente de rendre compte (la reconnaissance d'une société phuraliste) et l'idéologie à laquelle il fait référence (la description du Canada comme une mosaique et le sentiment d'identité et d'appartenance qui devrait en découler), idéologie porteuse d'un projet politique entrant en collision avec le projet québécois d'intégration des membres des communautés culturelles au sein d'une communauté nationale qui ne cache pas ses préférences (Labelle, Rocher et Rocher, 1995).

\section{Patriotisme constitutionnel et citoyenneté fonctionnelle}

Il est tentant de promouvoir une forme de "patriotisme constitutionnel» reposant sur la nécessité de dissocier les éléments pré-politiques de lidentité collective - la langue, la culture, l'histoire - du projet politique de la citoyenneté. Celui-ci définit l'espace commun sur la base des principes universels du 
libéralisme et de la notion d'État de droit. Une identité collective supra-nationale pourrait être édifiée sur une pratique de la citoyenneté découlant des concepts de l'autodétermination et de la souveraineté populaire. Le consensus nécessaire à cette pratique passe par l'acceptation du fait que la communauté politique est le fruit d'une association de citoyens libres et égaux. Cette pratique n'est finalement possible que si tous acceptent de se plier à un certain nombre de procédures. L'association des citoyens, qui détermine l'identité politique collective, se fonde sur une reconnaissance réciproque, sur la certitude que tous les citoyens peuvent espérer être considérés par tous comme libres et égaux.

Le patriotisme constitutionnel repose donc sur une culture politique commune, et non sur une culture ethnique ou nationale commune (Gamberale, 1997, 39). Prenant la Suisse et les États-Unis comme exemples, Habermas (1994) avance l'idée selon laquelle les principes constitutionnels ne doivent pas être fondés sur le fait que tous les citoyens partagent une même langue ou les mêmes origines ethniques et culturelles. La culture politique s'édifie sur une pratique commune de la citoyenneté, comprise comme l'ensemble des activités des individus dans l'espace public, où tous et chacun peuvent s'exprimer à la première personne du pluriel. Ainsi, le citoyen ne se définit pas par son appartenance à une communauté, ce qui relève de l'identité, mais plutôt par ses interventions dans l'espace public, lesquelles sont liées aux interventions des autres citoyens. Il soutient que la culture politique doit servir de «common denominator for a constitutional patriotism which simultaneously sharpens an awareness of the multiplicity and integrity of the different forms of life which coexist in a multicultural societyn (1995, 264). Les traditions nationales doivent être au diapason d'une culture politique partagée, et ce, au niveau supranational. Dans cette perspective, Habermas affirme qu'appliquée au cas de la mise en place d'une citoyenneté européenne, la culture politique partagée : 
[...] can today only be enacted in the paradoxical sense of compliance with the procedural rationality of a political willformation, the more of less discursive character of which depends on the vitality of the informal circuit of public communication. An inclusive public sphere cannot be organized as a whole; it depends rather on the stabilizing context of a liberal and egalitarian political culture. At the same time, such a communicative pluralism would still be ineffective unless further conditions could be met. In the first place, deliberations within the decision-making bodies should be open for and sensitive to the influx of issues, values, orientations, contributions, and programs originating from their informal environments. Only if such an interplay between institutionalized processes of opinion and will-formation and those of informal networks of public communication occurs can citizenship today mean more than the aggregation of prepolitical individual interests and the passive enjoyment of rights bestowed upon the individual by the paternalitic authority of the state (1995, 269-270).

Le projet de constitution d'une citoyenneté européenne admet des interprétations différenciées des mêmes droits universels et des principes constitutionnels qui sont déterminés par le contexte des différentes histoires nationales. Dans cette perspective, la citoyenneté européenne repose sur la capacité des États à partager une culture politique similaire tout en aménageant leur espace intérieur en fonction de leurs propres sensibilités. $\mathrm{La}$ citoyenneté commune et le patriotisme constitutionnel qui en découle ne sont pas incompatibles avec la notion de nationalisme libéral dont nous avons fait état plus haut. Le mérite d'Habermas est d'avoir dissocié les notions d'identité et de citoyenneté. Toutefois, cette approche semble davantage compatible avec le projet de construction de l'Union européenne et l'on peut se demander dans quelle mesure elle vaut tout autant dans les États multinationaux. Par ailleurs, si 
le patriotisme constitutionnel peut servir de modèle dans les États polyethniques, on peut douter qu'il soit suffisant dans les États multinationaux. Lorsqu'un État est à la fois multinational et polyethnique, comme c'est le cas notamment du Canada, lorsqu'il compte des uminorités nationales» territorialement constituées disposant d'institutions politiques qui leur sont propres (comme c'est le cas pour le Québec, et semble en voie de se réaliser pour plusieurs nations autochtones), l'exercice de la citoyenneté doit aussi prendre en compte le désir de pérennité de ces groupes.

Lorsque la citoyenneté est définie exclusivement en termes politiques, comme l'ensemble des droits et des responsabilités garantis et sanctionnés par le droit, elle est nécessairement unique. Ainsi, la loyauté ne peut être divisée. Le projet de constitution d'une citoyenneté à plusieurs niveaux, comme le prévoit le projet d'une citoyenneté européenne, pose donc problème. C'est pourquoi les tenants d'une citoyenneté qualifiée de post-nationale sont préoccupés par le fait qu'une dissociation entre identité nationale et citoyenneté puisse menacer la cohésion sociale et les pratiques démocratiques, et que l'intégration sociale ne devienne que fonctionnelle. Schnapper $(1997,209)$ rappelle que, pour pallier ce déséquilibre, certains ont proposé, dans le cadre de l'Union européenne, la conclusion d'un "contrat de citoyenneté» en vertu duquel les droits de la citoyenneté seraient attribués aux «étrangers nationaux» à la condition que ceux-ci s'engagent à adopter les valeurs démocratiques et à soutenir les lois nationales en ce qui a trait au respect des droits humains. De la même manière, le patriotisme constitutionnel d'Habermas implique que, même si tous ne parlent pas la même langue, tous partagent au moins un même langage (du moins dans l'acception des notions pertinentes au débat public), une même culture politique, les mêmes valeurs. Si tel n'est pas le cas, il est impossible de vouloir établir un espace de dialogue et de négociations, condition préalable aux pratiques démocratiques. 
En termes simples, le dilemme est le suivant : une société civile, établie sur des principes abstraits, comme le respect des droits humains et l'État de droit, peut-elle se substituer à la mobilisation à la fois affective et politique provoquée par l'appropriation de la tradition politique et culturelle de la nation? Pour Schnapper, "[t]he abstract or concrete aspect of the nation, let alone the concrete aspect of belonging to an ethnic group, will stir people more than the purely abstract notions of class consciousness, state of law, or buman rights (1997, 212). Ces réticences sont compréhensibles. Elles s'inscrivent toutefois dans une fausse dichotomie opposant société abstraite et société concrète. L'enjeu n'est pas de faire disparaittre la nation au profit de la citoyenneté, mais plutôt de réconcilier l'appartenance à des espaces discursifs différents, des lieux de dialogue qui ne se limitent pas aux intérêts véhiculés par un groupe national spécifique qui, le plus souvent, se trouve être le groupe national dominant. Schnapper peut difficilement concevoir qu'un État puisse être autre chose qu'un État-nation. Elle affirme que la participation à une société nationale repose concrètement sur un ensemble d'éléments pouvant être qualifiés d'ethniques : l'utilisation d'une langue commune, une culture commune, une mémoire historique particulière partagée par les membres de la nation, une participation au sein des mêmes institutions (et notamment le système scolaire, les lieux de travail). Ainsi, "[t] he immediate familiarity that exists between nationals, whatever other differences keep them apart, is the product of this specific socialization and the common life within a concrete national societyn $(1997,214)$. Ce faisant, les individus sont naturellement attachés aux éléments qui composent leur environnement familier, au sein duquel ils ont construit leur identité individuelle en relation avec leur identité collective. Dans cette perspective, la nation est tout à la fois une communauté de culture, un lieu où s'expriment une mémoire collective, une identité enracinée dans l'histoire et un projet civique. Pour Schnapper, la citoyenneté multinationale ne peut être appréhendée que dans le cadre de l'État-nation et sa portée doit être limitée : 
Allegiance to a political community must by nature remain distinct from the participation in the concrete society. [...] Multinational citizenship should only concern marginal groups, for the loyalty to a political onganization cannot be split; a double or triple allegiance would always inevitably lead to a loyalty conflict $(1997,216)$.

Cette auteure est d'abord et avant tout préoccupée par le maintien de liens concrets entre les membres de la société. La citoyenneté est appréhendée comme un outil d'intégration sociale. Les droits des citoyens visent à garantir la dignité de ceux-ci ainsi que les conditions concrètes permettant l'exercice de ces droits. Toutefois, la nature juridique et abstraite des droits est perçue comme étant insuffisante pour unir les citoyens. La citoyenneté exige aussi un engagement minimal à l'endroit de valeurs et de pratiques communes. Ainsi, une «nation démocratique» ne peut être que de nature civique, puisque le lien entre la nation et la citoyenneté relève moins de la logique que de l'histoire. Les sociétés ne peuvent être réduites aux seuls intérêts matériels.

La réflexion de Schnapper s'inscrit dans la logique de l'Étatnation et, plus particulièrement de l'État de type unitaire. Son approche constitue toutefois un plaidoyer en faveur de la reconnaissance de la nation comme lieu d'ancrage de la citoyenneté. Son analyse est cependant problématique pour les États qui sont déjà constitués sur la base du multinationalisme, là où il n'existe pas une mais plusieurs langues, là où les cultures (tout en partageant des valeurs communes) reposent sur des histoires et des mémoires collectives différenciées, là où les nations contrôlent des institutions politiques, économiques et sociales parallèlement à une ou plusieurs nations, ou en complémentarité ou en conflit avec elle(s). 


\section{État multinational et citoyenneté fonctionnelle Le cas canadien}

Le Canada, bien qu'État multinational, compte des nations aux statuts social et politique fort différent. La minorité nationale composée des francophones est, pour l'essentiel, concentrée au Québec. Plus de 81 p. 100 des Québécois possèdent le français comme langue maternelle et plus de $85 \mathrm{p}$. 100 de tous les individus ayant le français comme langue maternelle résident au Québec. Les minorités linguistiques francophones sont surtout concentrées au Nouveau-Brunswick (243 690 personnes) et en Ontario (503 345) et subissent, bien qu'à des degrés divers, le phénomène de l'assimilation : dans le premier cas, 91,6 p. 100 des francophones parlent toujours cette langue dans leur foyer, alors que dans le second cas, cette proportion tombe à 63,3 p. cent (McRoberts, 1998, 207-209). Les nations autochtones sont plus diversifiées et plus dispersées sur le territoire canadien. Selon le recensement de 1991, les peuples autochtones représentent environ 3,7 p. 100 de la population canadienne. Phus de la moitié d'entre eux possèdent le statut d'indien et sont affiliés à l'une des 605 bandes réparties entre les 2597 réserves du Canada (Fleras et Elliott, 1996). Pour que l'État canadien puisse fonctionner comme État multinational, il faudrait aussi que le groupe majoritaire se considère également comme une nation. Tel n'est pas le cas au Canada. Le déclin de l'approche dualiste, pourtant au cœur des débats politiques des années 1960, s'explique par les politiques "canadianisantes» adoptées par le gouvernement fédéral dès la fin des années 1960. Les politiques du bilinguisme officiel, du multiculturalisme ainsi que l'enchâssement d'une Charte des droits et libertés dans la nouvelle constitution de 1982 ont plutôt renforcé une politique de la diversité basée sur des identités nonterritorialement constituées, alimentant du même coup un courant de pensée déjà peu favorable à l'endroit de la 
reconnaissance de la "nation québécoise» et, plus globalement, de toute forme de nationalisme (Rocher et Smith, 1997).

Si le Canada doit être considéré comme un État multinational, l'appartenance nationale ne coïncide pas nécessairement avec la citoyenneté politique, et des rapports conflictuels existent entre ces deux éléments. Si le fédéralisme constitue l'une des formes politiques les mieux à même de reconnaître la diversité, l'expérience canadienne démontre que tel n'est pas toujours le cas (Gagnon et Rocher, 1997). Kymlicka explique cette tension, en s'inspirant des travaux de Resnick (1994), par le fait que le Québec et le reste du Canada ont adopté des visions différentes du fédéralisme : «l]e premier met l'accent sur le lien entre le fédéralisme et l'autonomie des minorités nationales, tandis que le second escamote ou atténue ce liens, $(1998,22)$. Ce second modèle, appelé fédéralisme territorial, repose sur les principes de l'égalité absolue des provinces et des citoyens. Il ne peut concevoir qu'une province puisse obtenir un «statut particulien» ni envisager une distribution asymétrique des pouvoirs afin de renforcer la capacité de la minorité nationale de se gouverner en fonction de ses propres priorités. Dans le reste du Canada, le fédéralisme est fondé sur le concept de régions, ces demières étant moins portées à vouloir protéger leur autonomie et limiter l'intervention du gouvernement central (le lieu d'expression politique des intérêts de la nation majoritaire) lorsque le bien-être des citoyens peut être mieux garanti par les politiques publiques adoptées par ce palier de gouvemement. C'est au nom de la perpétuation des normes et valeurs qui fondent leur identité nationale que les Canadiens anglais acceptent l'intervention du gouvernement fédéral dans des champs de compétence provinciale et consentent à ce que l'autonomie provinciale soit de plus en plus restreinte. Tout comme les Québécois, ils agissent en fonction des intérêts de leur nation. Ceux-ci s'expriment davantage à travers le gouvernement fédéral que par la somme des interventions provinciales. 
Pour le Québec, les conflits plongent leurs racines dans une compréhension multinationale du Canada et opposent donc deux projets nationaux qui privilégient parfois des modes d'intervention politique allant dans des directions différentes. Pour sa part, Jane Jenson parle de deux régimes de citoyenneté qui ne perçoivent pas de la même manière deux dimensions inhérentes à la citoyenneté, à savoir l'aspect droit et l'aspect appartenance (Jenson, 1998, 239)2.

Un fédéralisme fondé sur la cœxistence de nations implique l'établissement d'un rapport égalitaire, reposant sur des identités non hiérarchisées, entre ses différentes composantes nationales (Rocher et Sarra-Boumet, 1995). Un fédéralisme fondé sur les régions repose davantage sur un rapport de nature tutélaire, le gouvemement central se faisant le garant du bien-être de la «nation». Les dernières propositions de réforme de la fédération canadienne, qui trouvent leur plus récente expression dans la Déclaration de Calgary, vont dans ce sens (Rocher, 1998). Le principe de l'égalité des provinces ou des régions sert à masquer, selon Kymlicka, le principe de la nationalité. Les Canadiens anglophones utilisent le principe d'égalité pour justifier l'établissement d'une identité commune et consolider leur conception de la «nation canadienne». D'où leur incapacité à comprendre les revendications québécoises et à accepter tout aménagement institutionnel sur la base de l'asymétrie. Ainsi,

2 Jane Jenson inclut dans le concept de régime de citoyenneté les éléments suivants : whe institutional arrangements, rules and understandings that guide and shape state policy; problem definition employed by states and citizens; and the range of claims recognized as legitimater $(1997,631)$. La modernisation politique, sociale et économique qui a marqué l'évolution du Québec depuis les années 1960 a fait en sorte que les aménagements institutionnels (qu'ils soient privés ou publics) ont contribué à promouvoir la communauté nationale québécoise, parfois contre, parfois en accord avec les politiques du gouvernement fédéral. Voir aussi Jenson (1995). 
leur refus de reconnaître le Québec comme nation sert de paravent à un autre nationalisme d'autant plus pernicieux qu'il s'ignore (Resnick, 1995), mais qui sert tout de même à mettre de l'avant une conception anglo-canadienne de la nationalité unique. Qui plus est, et il s'agit ici d'un élément fondamental de l'équation, cette approche s'inspire d'un modèle jacobin qui ne peut concevoir le développement politique en dehors du processus d'homogénéisation des populations (et des nations) par la constitution d'une pyramide de pouvoirs hiérarchisés dans le cadre d'un État-nation unitaire chapeautant l'ensemble de la société (Théret, 1995, 14).

Dans un État multinational, contrairement aux craintes avancées par Schnapper, il est souhaitable de recomposer le rapport entre identité nationale et citoyenneté sur des bases qui s'éloignent du modèle de l'État unitaire. La citoyenneté doit reposer sur des assises concrètes et servir d'outil d'intégration sociale, tout en reconnaissant qu'elle puisse varier d'un espace national à un autre au sein d'un même État, d'une même communauté politique. Un État multinational doit être ouvert à des aménagements politiques particuliers en regard de la citoyenneté sans pour autant nier le principe de l'égalité des personnes. Pour Kymlicka :

En démocratie libérale, certes, on doit respecter rigoureusement le principe de l'égalité morale entre les personnes et accorder un égal soin aux intérêts de toutes. Mais l'égalité entre les citoyens n'exige pas qu'on accorde des pouvoirs égaux à toutes les composantes d'une fédération. Au contraire, la reconnaissance d'un statut asymétrique pour des unités fondées sur la nationalité peut en fin de compte servir plus efficacement l'égalité morale, puisqu'elle apporte aux minorités le même souci et le même respect qu'à la nation majoritaire. [...] Un fédéralisme asymétrique qui prend en compte ces différentes identités n'implique donc à l'égard des 
individus aucun manque de respect ni aucune discrimination $(1998,26-27)$.

Dans un État multinational, et a fortiori dans un régime fédéral, la légitimité du gouvernement central est d'abord et avant tout fonctionnelle. Si tel n'est pas le cas au Canada, c'est parce qu'il y a osmose entre identité nationale et citoyenneté "canadienne» qui, du fait de l'histoire, trouve ses assises dans un État qui est aussi fédéral. L'attachement manifesté à l'endroit du "pays" au Canada anglais s'oppose profondément à toute reconnaissance formelle du multinationalisme où même du nationalisme lui-même, en dépit du fait que l'importance donnée au discours sur le "pays» est, en elle-même, profondément nationaliste. De la même manière, il est possible d'envisager un régime de citoyenneté qui s'inspire des principes fédéraux et, plus particulièrement, de ceux qui découlent du besoin de sauvegarder la qualité propre de chaque minorité nationale, du respect de l'altérité, de la nécessité de s'opposer à toute simplification totalitaire et à toute uniformisation imposée par le pouvoir central selon un modèle jacobin de gouverne politique (Sidjanski, 1994, 73). En fait, dans un État fédéral, la loyauté ne peut être que multiple (Vernon, 1988). En d'autres termes, il faut aussi opérer une distinction entre le fondement fonctionnel de l'État multinational / fédéral dont la légitimité devrait reposer sur son efficacité à prendre en compte les problèmes qui relèvent de ses compétences et le fondement national des États (nationaux) fédérés dont la légitimité devrait relever de leur capacité à protéger la spécificité des communautés historiques qu'ils abritent. Pour reprendre la formule de Théret : «à l'État fédéral la protection de la société, à l'état fédéré la protection de la communauté» $(1995,322)$. La citoyenneté devrait être appréhendée suivant la même logique. La citoyenneté dans un État multinational / fédéral ne peut être que fonctionnellement construite. Cette approche permet de répondre aux peurs de Schnapper pour qui la nation est le lieu 
d'ancrage des solidarités concrètes, tout en permettant de prendre en compte le modèle d'Habermas d'un patriotisme constitutionnel, qui renvoie à des valeurs qui sont tout à la fois universelles et respectueuses des différences. La loyauté à l'endroit de la nation, fondement de l'identité individuelle et collective, n'entre plus en conflit avec la loyauté que les citoyens doivent porter au niveau fédéral si, et seulement si, celui-ci ne cherche pas à hiérarchiser les identités et imposer un pouvoir tutélaire aux minorités nationales.

Dans un État multinational, la meilleure façon de promouvoir un sens de la solidarité passe par l'accommodement des identités nationales phutôt que par leur subordination. Comme le rappelle Kymlicka, "[p]eople from different national groups will only share an allegiance to the larger polity if they see it as the context within which their national identity is nurtured, rather than subordinateds (1995a, 189). Il mentionne que, dans les pays qui sont à la fois polyethniques et multinationaux, les groupes culturels sont non seulement différents, mais véhiculent des images différentes du pays. Cela signifie que ses membres doivent chérir la diversité et aussi respecter la diversité des approches de la diversité. Est-ce suffisant pour que les citoyens aux identités nationales singulières veuillent continuer à partager le même espace politique? Il n'y a pas de réponse universelle à cette question. Le processus est sûrement plus laborieux dans le cas de la création de nouveaux ensembles politiques, comme le démontrent les tergiversations autour de la signification à donner à la citoyenneté européenne. Dans le cas des pays existants, la loyauté est en partie conditionnée par la profondeur de la reconnaissance de la diversité. Le poids de l'histoire constitue un autre facteur non négligeable. Par exemple, un récent sondage démontrait que, parmi les francophones québécois, 14 p. 100 se définissaient uniquement comme Québécois et à peine 3 p. 100 uniquement comme Canadien. Pour le plus grand nombre, l'identité est multiple : 31 p. 100 considèrent que leur identité est canadienne et 
québécoise à part égale, 37 p. 100 se définissent d'abord comme Québécois mais aussi comme Canadiens, 13 p. 100 affirment qu'ils sont d'abord Canadiens puis ensuite Québécois (Wells, 1998).

Cette citoyenneté fonctionnelle se distingue de ce que Meehan (1993) a appelé la "citoyenneté multiple». La construction de l'Union européenne, dominée par la logique économique conduisant à la constitution d'un marché commun, d'une monnaie commune, d'une politique économique intégrée à laquelle les États doivent se conformer, ne peut aboutir qu'à une intégration politique complète. Ce faisant, la citoyenneté classique, qui s'articule autour des droits politiques, devient moins importante, au profit des garanties concernant la pleine participation économique des individus. La citoyenneté peut donc s'exercer à différents niveaux. Cette conception de la société tend à atténuer le poids de la communauté politique (lire ici État-nation) et historique et remplace cette dernière par celle de l'organisation de la production et de la redistribution de la richesse. Pour Schnapper, «[p]artners are no longer related by $a$ contract of political nature but by their common participation in the social and economic lifen $(1997,208)$. La notion de citoyenneté fonctionnelle permet d'éviter cet écueil en insistant sur l'importance de la communauté nationale comme lieu d'intégration sociale, tout en reconnaissant qu'au sein des sociétés multinationales il est possible, sinon souhaitable, que certaines activités soient dévolues ou partagées avec un autre palier de gouvemement. Si les responsabilités relevant de chaque palier de gouvernement sont clairement établies et respectées, la citoyenneté ne perd pas ses attributs politiques. Elle se conjugue plutôt dans un espace et une histoire donnés tout en s'élargissant par son insertion dans un plus grand ensemble. C'est du moins le modèle qui devrait découler d'une reconnaissance du caractère multinational du Canada et permettre des aménagements différenciés de la citoyenneté, ce 
qui autoriserait le Québec à inscrire sa propre lecture de la citoyenneté dans le cadre de l'ensemble canadien.

\section{Citoyenneté fonctionnelle et pluralité juridique}

Une fois reconnu et accepté le principe de la citoyenneté fonctionnelle, se pose la question de son opérationalisation. Celle-ci n'est possible que si les communautés nationales partagent les mêmes valeurs et le même attachement aux principes du libéralisme démocratique. Cette condition, incontoumable, ne doit pas être confondue avec le fait qu'il puisse y avoir des identités nationales particulières. L'intention du gouvernement fédéral canadien au cours des trois demières décennies était justement de promouvoir l'unité dite "nationale» (lire pan-canadienne) sur la base de valeurs communes et, ce faisant, diminuer les tensions avec le Québec ${ }^{3}$. Norman fait remarquer, à juste titre, que :

[...] shared values or principles are neither necessary nor sufficient for national unity of the sort relevant in the Canadian context. Whether or not Canada should remain united is, at

3 Cette façon de voir fut au cœur du débat constitutionnel qui a marqué la politique canadienne au cours des dix demières années. Elle constituait la trame de fond de tous les documents officiels, que ce soit le Forum des citoyens sur l'avenir du Canada (1991a) ou les intentions fédérales en matière de renouvellement de la constitution (Canada 1991b, 1991c). Cette approche continue de dominer le discours public. Par exemple, Jean Charest, ancien chef du Parti progressiste conservateur du Canada, œuvrant maintenant sur la scène politique québécoise, veut persuader les Québécois que les Canadiens partagent les mêmes valeurs qu'eux et que, sur cette base, les tensions entre le Québec et le Canada peuvent aisément être atténuées (Cloutier, 1998, A-1). 
bottom, a question of why two or more polities should want to share the same country. My argument is simply that the ideology of shared values is, by itself, a hollow and disappointing answer to that question. $(1995,141)$

Si le fait de partager les mêmes valeurs ne suffit pas à justifier le fait d'appartenir à une même entité politique (sur cette seule base, le Canada pourrait s'annexer aux Etats-Unis, la Suède et la Norvège pourraient à nouveau se réunir), cela semble essentiel pour pouvoir appréhender de manière relativement cohérente les droits fondamentaux. En d'autres termes, les droits et les obligations associés à la citoyenneté ont besoin d'être soutenus par une identité collective commune (qui est portée par la nation), mais ils doivent aussi être compatibles avec la façon dont ils sont compris au sein d'une organisation politique plus large (la fédération canadienne ou l'Union européenne), justement pour qu'existe une identité politique commune. Cette distinction n'est pas artificielle. La première n'est pas réductible à la seconde, elle se doit plutôt d'être compatible avec elle.

Schneiderman proposait récemment un modèle permettant d'aménager un régime de pluralité juridique en fonction des identités collectives particulières. Ce juriste part de l'idée qu'il existe un umétadroib), lui-même générateur de droits particuliers $(1998,160)$. Il rompt aussi avec le modèle juridique jacobin et admet que les divergences en matière de droits proviennent d'appartenances à des communautés nationales différentes. Il reconnaît que le Canada possède des identités nationales distinctes que le régime politique fédéral doit réconcilier, et non d'éradiquer. L'atteinte d'un consensus sur la constitutionnalisation des droits de la personne repose moins sur un accord global sur les normes abstraites qui définissent ces droits que sur ala participation active et le consentement formel de toutes les composantes de la société». Il ajoute que "[c]ette 
recherche ne répond pas seulement à un principe de base du libéralisme démocratique; elle est aussi un moyen d'atteindre, entre les diverses cultures, un consensus sur les droits humains" $(1998,179-180)$.

Se fondant sur le fait qu'il existe une très grande compatibilité entre la Charte canadienne des droits et libertés et la Charte des droits et libertés de la personne (du Québec), lune et l'autre respectant les principes du libéralisme et ses exigences juridiques, il suggère d'adopter un régime pluraliste fondé sur le respect et le consentement mutuels, où chaque groupe pourrait jouir de la marge de mancuvre nécessaire pour observer son propre code.

Les deux chartes divergent toutefois au chapitre des régimes délimitant les droits linguistiques. La Charte canadienne, et plus spécifiquement l'article 23 , établit des normes pancanadiennes quant aux droits de la minorité linguistique à l'enseignement dans sa langue. Prenant sa source dans la Loi sur les langues officielles (LLO) adoptée en 1969 par le gouvernement fédéral, qui établissait l'anglais et le français comme les langues officielles et donnait aux citoyens le droit d'obtenir des services gouvernementaux dans l'une ou l'autre langue à l'échelle du pays (là où le nombre le justifie), la Charte est venue confirmer le statut symétrique des deux langues : les minorités francophones hors Québec et anglophone au Québec sont traitées de la même manière. De plus, elle s'inspire d'une approche fondée sur les droits individuels dans la mesure où les individus possèdent les mêmes droits d'un bout à l'autre du pays. Ainsi, la LLO et la Charte n'abordent pas les droits linguistiques en fonction de leur ancrage territorial ou en fonction de l'environnement démolinguistique dans lequel elles évoluent.

Le Québec a adopté son propre régime d'aménagement linguistique (Charte de la langue franfaise - CLF), qui faisait du français la langue officielle de l'État, favorisait la francisation des 
lieux de travail et de l'espace public (notamment en matière d'affichage et de langue des affaires) et dirigeait les enfants autres que ceux de la minorité anglophone du Québec vers le réseau des écoles françaises pour l'enseignement au primaire et au secondaire (Coulombe, 1998). Plusieurs dispositions de la CLF ont été contestées avec succès devant les tribunaux qui ont déclaré ultra vires les dispositions qui faisaient du français la langue officielle de l'État et des tribunaux, qui dirigeaient les enfants anglophones des autres provinces canadiennes vers les écoles françaises, ou faisaient du français la seule langue de l'affichage commercial (à l'exception des activités à caractère culturel et religieux). La Charte canadienne fut articulée autour de la consolidation du régime des droits linguistiques tel qu'il avait été défini par le gouvernement fédéral. On trouve la démonstration de cette intention dans le fait que les dispositions de la Charte en matière de droits linguistiques ne peuvent être contournées par l'utilisation de la clause dérogatoire de la Charte, alors que les libertés fondamentales, les garanties juridiques et les droits à l'égalité, pourtant au cœur des droits fondamentaux qui définissent la citoyenneté, y sont sujets. C'est dire qu'il existe une hiérarchie explicite dans la Charte canadienne entre les droits linguistiques et les libertés fondamentales, les premiers étant inamovibles. Alors que certains plaident en faveur de la reconnaissance de la légitimité des deux régimes linguistiques (Coulombe, 1998), d'autres ont plutôt souligné qu'ils s'inscrivent dans des projets politiques contradictoires, le régime canadien s'opposant au projet québécois de faire du français la langue publique commune (Labelle, Rocher et Rocher, 1995).

L'établissement d'un régime de pluralité juridique devrait contribuer à dénouer cette impasse. Schneiderman propose de revoir la clause 23 de la Charte canadienne et d'en retirer les éléments ayant invalidé certaines dispositions de la CLF. Il reprend à son compte la suggestion formulée entre autres par Woehrling (Morin et Woehrling, 1994, 245 ; voir aussi Cardinal 
et Thériault, 1992), qui mettait de l'avant la notion de réciprocité : ouvrir les écoles publiques de langue anglaise aux résidents des autres provinces si ces demières fournissent à leur minorité francophone des services éducatifs de même nature et de même niveau.

Par ailleurs, cette approche fonctionnelle des régimes juridiques pourrait prendre trois formes : soit ne pas appliquer au Québec la Charte canadienne et remodeler la Charte québécoise en lui retirant "certaines dispositions qui lui donnent l'allure d'un code provincial des droits humains (par exemple, les droits qui s'appliquent non seulement au gouvernement, mais aussi à l'action (privée») et d'en faire une loi distincte» (Schneiderman, $1998,184)$; soit instaurer un régime dualiste où la Charte québécoise aurait préséance quand il s'agira de lois québécoises, tandis que la Charte canadienne s'appliquerait seulement aux lois fédérales et aux lois des autres provinces; soit reconnaitre la complémentarité des deux chartes tout en admettant la préséance de la Charte canadienne - dans un tel cas, on pourrait instituer un nouveau tribunal constitutionnel comparable aux cours constitutionnelles que l'on trouve dans plusieurs pays d'Europe (1998, 184-187).

Il n'entre pas dans le cadre de cet article de déterminer quel modèle serait le mieux à même d'articuler convenablement les droits associés à la citoyenneté fonctionnelle. Néanmoins, ces trois hypothèses reposent sur quatre principes fondamentaux : 1) la reconnaissance du caractère multinational du Canada; 2) la compatibilité des valeurs qui rendent possible la constitution d'une identité politique commune; 3) l'absence de hiérarchisation des identités entre les diverses loyautés professées par les citoyens; 4) la légitimité d'établir des régimes juridiques différenciés dans un même État (fédéral). 
Ce modèle de citoyenneté fonctionnelle permet de voir comment les droits peuvent être aménagés dans une société multinationale et comment le Québec pourrait se dissocier en partie du cadre juridique jacobin dominant au Canada, cadre chapeauté par la Charte canadienne des droits et libertés. Il n'indique pas de quelle manière ces droits doivent s'articuler en prenant en compte le caractère polyethnique de ces communautés. Reste ouvert le débat concemant les aménagements particuliers devant faire en sorte que la citoyenneté, comprise non seulement en termes de droits abstraits et universels mais aussi en fonction de la capacité des citoyens d'exercer leurs droits, puisse être un vecteur d'inclusion. Gamberale souligne que l'exclusion de la citoyenneté peut prendre plusieurs formes. L'exclusion formelle découle d'une distinction entre les citoyens et les autres, et elle est généralement dirigée vers les membres des autres collectivités nationales. On peut aussi parler d'exclusion matérielle lorsque certains groupes d'individus (les minorités ethniques, les gais et lesbiennes, les femmes, les handicapés, les exclus du marché du travail), en dépit de leur insertion dans la communauté, ne disposent pas des moyens d'exercer leurs droits de citoyens à cause de leur statut économique et social $(1997,53)$. Iris Marion Young résume bien le problème lorsqu'elle affirme que :

[...] the inclusion and participation of everyone in public discussion and decision making requires mechanisms for group representation. [...] where differences in capacities, cultures, values, and behavioral styles exist among groups, but some of these groups are privileged, strict adherence to a principle of equal treatment tends to perpetuate oppression or disadvantage. The inclusion and participation of everyone in social and political institutions therefore sometimes requires the articulation of special rights that attend to group differences in order to undermine oppression and disadvantage. $(1995,177)$ 
Pour que la citoyenneté devienne effective, la délibération publique doit être réelle. Les espaces de délibération doivent être accessibles et ouverts à une phuralité de points de vue, les institutions politiques, sociales, culturelles et économiques doivent refléter l'hétérogénéité de la société qu'elles sont censées servir et représenter.

Si la citoyenneté fonctionnelle permet de réintroduire les identités intermédiaires entre le citoyen et l'État, dimension négligée ou tout simplement mise de côté par le libéralisme classique, il n'en demeure pas moins que la pratique étatique ne doit pas être alimentée par le désir de préserver une seule forme d'allégeance communautaire. On ne saurait donc, au nom de la préservation de la communauté nationale, faire fi des principes universels. En ce sens, on ne peut qu'être d'accord avec Habermas lorsqu'il soutient que "[t] he identity of a political community [...] depends primarily upon constitutional principles rooted in a political culture and not upon ethnic-cultural form of life as a wholes (1995, 278). D'où l'importance d'une vigilance constante à l'endroit des chartes qui doivent s'inscrite dans la tradition démocratique libérale. La citoyenneté fonctionnelle n'est pas la consécration du principe national, elle n'est que le lieu d'ancrage où doivent s'articuler les droits universels. Elle permet seulement de résoudre le dilemme entre l'abstrait et le concret. La citoyenneté n'est, après tout, qu'un construit historique. On ne saurait donc évacuer l'histoire de la citoyenneté. 
REVUE INTERNATIONALE D'ÉTUDES QUÉBÉCOISES

\section{Bibliographie}

Beiner, Ronald. 1992. What's the Matter with Liberalism? Berkeley and Los Angeles : University of Califomia Press.

Beiner, Ronald. 1995. "Why Citizenship Constitutes a Theoretical Problem in the Last Decade of the Twentieth Centuryn, Theorizing Citizenship, publié par R. Beiner. Albany : State University of New York Press, pp. 1-28.

Cahen, Michel. 1994. Ethnicité politique. Pour une lecture réaliste de l'identité. Paris : L'Harmattan.

Canada. 1991a. Le Forum des citoyens sur l'avenir du Canada. Rapport à la population et au gouvernement du Canada. Ottawa : Approvisionnements et Services Canada.

Canada. 1991b. Rapport du Comité mixte spécial de la Chambre des communes. Le processus de modification de la Constitution du Canada. Ottawa : Troisième session de la trente-quatrième législature.

Canada. 1991c. Bâtir ensemble l'avenir du Canada. Propositions. Ottawa : Approvisionnements et Services Canada.

Cardinal, Linda. et J. Yvon Thériault. 1992. «La francophonie canadienne et acadienne confrontée au défi québécois», Répliques aux détracteurs de la souveraineté du Québec, publié par A.-G. Gagnon et F. Rocher. Montréal : VLB éditeur, pp. 329-341.

Cloutier, Mario. 1998. "Québec et Canada, même combat. Jean Charest se présente comme un nationaliste pouvant démontrer que Canadiens et Québécois partagent les mêmes valeurs", Le Devoir, 6 avril, A-1 et A-8.

Coulombe, Pierre. 1998. «Language Rights and Federal Citizenship in Canada and Spain : Preliminary Thoughts». Communication présentée à la Conference In Search of Justice and Stability : Liberal Justice and Political Stability in Multinational Societies organisée par le Groupe de recherche sur les sociétés plurinationales, North Hatley, Québec, 27-29 mars 1998.

Fleras, Angie et Jean Leonard Elliott. 1996. Unequal Relations. An Introduction to Race, Ethnic and Aboriginal Dymamics in Canada, Scarborough : Prentice Hall. 
Gagnon, Alain-G. et François Rocher. 1997. «Nationalisme libéral et construction multinationale: la représentation de la "nation" dans la dynamique Québec-Canadas, Revue internationale d'études canadiennes, 16 (automne).

Gamberale, Carlo. 1997. «European Citizenship and Political Identityn, Space \& Polity, 1 (1), pp. 37-59.

Gellner, Emest. 1981. Nations and Nationalism. Oxford : Blackwell.

Habermas, Jürgen. 1994. «European Citizenship and National Identities", The Conditions of Citizenship, publié par B. Van Steenbergen. London : Sage, pp. 20-35.

Habermas, Jürgen. 1995. "Citizenship and National Identity : Some Reflections on the Future of Europen, Theorizing Citizenship, publié par R. Beiner. Albany : State University of New York Press, pp. 255-281.

Jenson, Jane. 1995. "Citizenship Claims : Routes to Representation in a Federal System", Rethinking Federalism. Citizens, Markets, and Governments in a Changing World, publié par K. Knop, S. Ostry, R. Simeon et K. Swinton. Vancouver : UBC Press, pp. 99118.

Jenson, Jane. 1998. «Reconnaitre les différences : Sociétés distinctes, régimes de citoyenneté, partenariat, Sortir de l'impasse. Les voies de la réconciliation, publié par $\mathrm{G}$. Laforest et $\mathrm{R}$. Gibbins. Montréal : Institut de recherches en politiques publiques, Pp. 235262.

Kymlicka, Will. 1995a. Multicultural Citizenship. A Liberal Theory of Minority Rights. Oxford: Oxford University Press.

Kymlicka, Will. 1995b. «Le libéralisme et la politisation de la culture», Une nation peut-elle se donner la constitution de son choix?, publié par M. Seymour. Montréal : Bellarmin, pp. 93-119.

Kymlicka, Will. 1998. «Le fédéralisme multinational au Canada : un partenariat à repensen,, Sortir de l'impasse. Les voies de la réconciliation, publié par $\mathrm{G}$. Laforest et $\mathrm{R}$. Gibbins. Montréal : Institut de recherches en politiques publiques, pp. 15-54.

Kymlicka, Will et Wayne Norman. 1995. «Return of the Citizen : A Survey of Recent Work on Citizenship Theoryn, Theorizing Citizenship, publié par R. Beiner. Albany : State University of New York Press, pp. 283-322. 
Labelle, Micheline, François Rocher et Guy Rocher. 1995. «Pluriethnicité, citoyenneté et intégration : de la souveraineté pour soulever les obstacles et les ambiguités", Cabiers de recherche sociologique, 25, pp. 213-245.

McRoberts, Kenneth. 1998. «Les minorités linguistiques dans un partenariat Canada-Québec", Sortir de l'impasse. Les voies de la réconciliation, publié par Guy Laforest et Roger Gibbins. Montréal : Institut de recherches en politiques publiques, pp. 203-234.

Meehan, Elizabeth. 1993. "Citizenship and the European Communityn, Political Quaterly, 64 (2) April-June, pp. 172-186.

Morin, Jacques-Yvan et José Woehrling. 1994. Demain le Québec : Choix politiques et constitutionnels d'un pays en devenir. Sillery : Septentrion.

Norman, Wayne. 1995. "The Ideology of Shared Values : A Myopic Vision of Unity in the Multi-nation State», Is Quebec Nationalism Just? Perspectives from Anglophone Canada, publié par J. H. Carens. Montréal : McGill-Queen's University Press, Pp. 137-159.

Resnick, Philip. 1994. «Toward a Multinational Federalism : Asymmetrical and Confederal Altematives", $\dot{A}$ la recherche $d^{\prime}$ un nouveau contrat politique : options asymétriques et options confédérales, publié par F. L. Seidle. Montréal : Institut de recherches en politiques publiques. pp. 71-89.

Rocher, François et Michel Sarra-Boumet. 1995. «La longue quête de l'égalité», Manifeste des intellectuels pour la souveraineté, suivi de Douze Essais sur l'avenir du Québec, publié par M. Sarra-Boumet. Montréal : Fides, pp. 43-57.

Rocher, François et Miriam Smith. 1997. «The New Boundaries of Canadian Political Culture",, Joumal of History and Politics,12 (2), pp. 36-70.

Rocher, François, avec la collaboration de André Lecours et Christian Rouillard. 1998. «Recognition Claims, Partisan Politics and Institutional Constraints: Belgium, Spain and Canada in a Comparative Perspective». Communication présentée à la Conference In Search of Justice and Stability : Liberal Justice and Political Stability in Multinational Societies organisée par le Groupe de recherche sur les sociétés plurinationales, North Hatley, Québec, 27-29 mars 1998.

Schneiderman, David 1998. «Droits humains, différences fondamentales? Vers un partenariat régi par plusieurs chartes", Sortir 
de l'impasse. Les voies de la réconciliation, publié par Guy Laforest et Roger Gibbins. Montréal : Institut de recherches en politiques publiques, pp. 159-202.

Schnapper, Dominique. 1997. "The European Debate on Citizenship", Daedalus,126 (3), pp. 199-222.

Sidjanski, Dusan. 1994. "Fédéralisme (et néo-fédéralisme)», Dictionnaire international du fédéralisme, publié par Denis De Rougernont. Bruxelles : Bruylant.

Tamir, Yael. 1993. Liberal nationalism. Princeton : Princeton University Press.

Théret, Bruno. 1995. Du fédéralisme et de la protection sociale en Amérique et en particulier au Canada, Rapport pour la Convention d'étude no 5 / 1995 du 4 juillet 1995. Paris : Commissariat Général du Plan.

Vernon, Richard. 1988. "The Federal Citizen», Perspectives on Canadian Federalism, publié par R. D. Olling et M. W. Westmacott. Scarborough : Prentice-Hall Canada, pp. 3-15.

Wells, Paul. 1998. «Quebecers? Canadians? We're proud to be bothy, The Montreal Gazette, 4th April.

Young, Iris Marion. 1995. «Polity and Group Difference : A Critique of the Ideal of Universal Citizenship», Theorizing Citizenship, publié par R. Beiner. Albany : State University of New York Press, pp. 175-207. 